\title{
Successful recovery from misdirection syndrome in nanophthalmic eyes by performing an anterior vitrectomy through the anterior chamber
}

\author{
Andi Akhmad Faisal • Muhammad Irfan Kamaruddin · Ryotaro Toda • \\ Yoshiaki Kiuchi $(\mathbb{D}$
}

Received: 5 July 2017/ Accepted: 15 December 2017 / Published online: 3 January 2018

(C) The Author(s) 2018. This article is an open access publication

\begin{abstract}
Purpose To determine the effectiveness of iridectomy, capsulotomy and anterior vitrectomy through the anterior chamber to treat misdirection syndrome in pseudophakic nanophthalmic eyes.

Methods This was a non-comparative study of seven nanophthalmic eyes from four consecutive patients. All eyes developed misdirection syndrome after successful cataract surgery. Treatment for misdirection syndrome involved capsulotomy and anterior vitrectomy through a peripheral iridectomy from the anterior chamber using a 25 -gauge vitreous cutter. The best-corrected visual acuity, intraocular pressure and anterior and posterior segment findings were recorded before and after surgery.

Results Resolution of the aqueous misdirection was achieved in all but one eye. The single case of recurrence was observed after a mean follow-up of $45.6 \pm 21.5$ months and was caused by closure of the
\end{abstract}

A. A. Faisal · M. I. Kamaruddin · R. Toda ·

Y. Kiuchi $(\square)$

Department of Ophthalmology and Visual Sciences,

Graduate School of Biomedical Sciences, Hiroshima

University, 1-2-3 Kasumi, Minami-ku,

Hiroshima 734-8551, Japan

e-mail: ykiuchi@hiroshima-u.ac.jp

A. A. Faisal · M. I. Kamaruddin

Department of Ophthalmology, Hasanuddin University,

Perintis Kemerdekaan Street 6th Kilometers,

Makassar City, South Sulawesi 90245, Indonesia capsule hole by Elschnig's pearls. This eye was successfully treated by enlargement of the lens capsule hole with a vitreous cutter. The mean intraocular pressure before surgery was $28.7 \pm 4.4 \mathrm{mmHg}$, and this was significantly reduced to $13.7 \pm 1.3 \mathrm{mmHg}$ at the final visit. All but one patient, who had uveal effusion, maintained their best-corrected visual acuity. Conclusion In this study, we investigated an alternative option for the treatment of misdirection syndrome in nanophthalmic eyes. We undertook a lens capsulotomy and anterior vitrectomy through a peripheral iridectomy from the anterior chamber using a 25-gauge vitreous cutter, which was able to create a communication hole between the anterior and posterior chambers.

Keywords Nanophthalmos · Angle-closure glaucoma $\cdot$ Misdirection syndrome $\cdot$ Lensectomy · Anterior vitrectomy

\section{Introduction}

Nanophthalmic eyes are characterized by an axial length of less than $20 \mathrm{~mm}$ and are associated with hyperopia. The degree of hyperopia is inversely correlated with the axial length [1]. Individuals with nanophthalmos are at high risk of developing angleclosure glaucoma [2,3], which is a consequence of 
having a shallow anterior chamber (AC) and a relatively large lens compared with the overall ocular volume [4].

Cataract extraction has been suggested as a treatment option for eyes with primary angle-closure diseases [5, 6]. However, cataract surgery in a nanophthalmic eye is technically difficult and is associated with an increased risk of complications $[1,7]$ including posterior capsular rupture, uveal effusion [8], choroidal hemorrhage, vitreous hemorrhage [9], retinal detachment and aqueous misdirection $[4,9,10]$.

Aqueous misdirection is a rare complication that is associated with an increase in the intraocular pressure (IOP) and a shallow or flat AC without a pupillary block or choroidal abnormality [11]. Aqueous misdirection syndrome develops after cataract surgery more frequently in nanophthalmic eyes than in eyes with a normal axial length [12]. A blockage of aqueous flow from the posterior chamber to the $\mathrm{AC}$ causes the misdirection syndrome, and treatments are focused on removing the cause of the blockage.

The purpose of this report is to present the efficacy and safety of our surgical approach for the management of misdirection syndrome in pseudophakic nanophthalmic eyes. The surgery consisted of peripheral iridectomy, creation of a hole in the peripheral lens capsule and an anterior vitrectomy through the AC.

\section{Methods}

\section{Participants}

This was a retrospective study of seven eyes from four patients who developed misdirection syndrome within a period of 1 day -9 months after cataract surgery.

We reviewed charts from the Hiroshima University Hospital between January 2009 and June 2014 and selected patients with nanophthalmos. Nanophthalmos was diagnosed based on an axial length of less than $20 \mathrm{~mm}$ and a shallow AC. Medical charts were reviewed for age at initial consultation, gender, bestcorrected visual acuity (BCVA), IOP, ocular findings by slit lamp and fundus ophthalmoscope. Family and surgical history, axial length, refractive error, surgical procedure and surgical complications were also recorded. Table 1 shows the background characteristics of the participants.

Surgical procedures

A micro-vitreoretinal blade was used to make an incision parallel to the iris plane in the peripheral cornea. Even though the patients had a high IOP as a result of the misdirection syndrome, there was a space between the iris and cornea. An AC infusion cannula was inserted through the incision to infuse balanced salt solution into the AC.

In a similar fashion, a second corneal incision was made at the site used for the original iridectomy or iridotomy and a 25-gauge vitreous cutter (Alcon Japan Ltd., Tokyo, Japan) was inserted into the AC. For eyes without a prior iridectomy, the 25-gauge vitreous cutter was used to create an iridectomy in the peripheral iris. The lens capsule was then pierced with a 25-gauge V-lance (Alcon Japan Ltd.), and the cutter was used to enlarge the opening in the lens capsule. This established a pathway between the AC and the vitreous cavity. Anterior vitrectomy was performed with a special focus on the vitreous immediately posterior to the intraocular lens (IOL) and near the lens capsule hole. After completion of the vitrectomy, a determination was made whether leakage was occurring at the self-sealing sites. If leakage was present, a suture was used to close the leakage site. The surgery was completed within $15 \mathrm{~min}$.

\section{Results}

The results are summarized in Table 1 . All but one of the eyes developed misdirection syndrome after an uneventful phacoemulsification and aspiration (PEA) and IOL implantation. One eye developed misdirection of the aqueous flow during removal of the residual cataract that remained after the lens was removed. Although surgery on the anterior vitreous and peripheral lens capsule resolved the aqueous misdirection in all seven eyes, three nanophthalmic eyes had high postoperative IOP that was caused by extensive peripheral anterior synechia, even after resolution of the misdirection syndrome. One eye had uveal effusion after recovering from misdirection syndrome. 


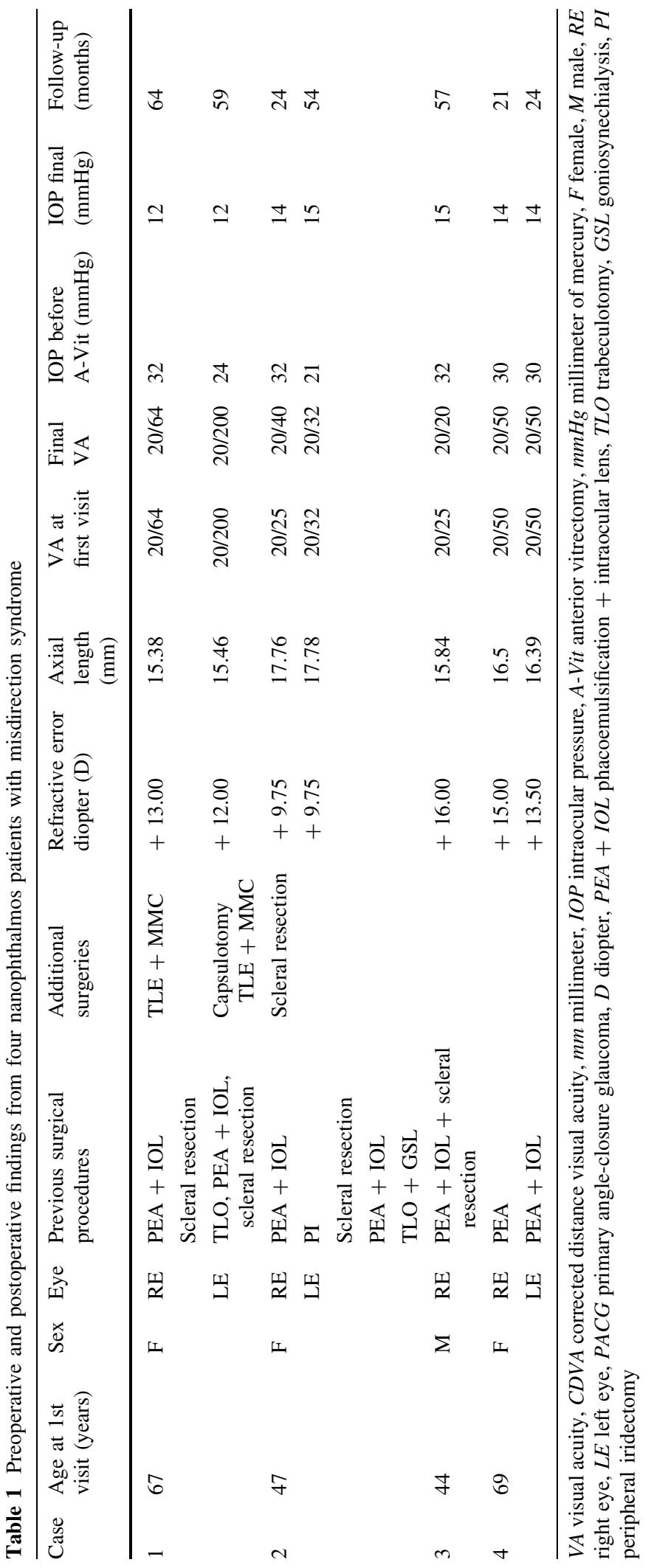


Case reports

Case 1 A 67-year-old woman with a history of bilateral primary angle-closure glaucoma was referred to the Hiroshima University Hospital in June 2009.

Her BCVA at the first visit was 20/64 with a refractive correction of +13.00 spherical diopters (D) for the right eye and 20/200 with +12.00 spherical D for the left eye. Slit-lamp biomicroscopy revealed that the AC was shallow in both eyes and measurements with the IOLMaster optical biometer (Carl Zeiss Meditec AG, Jena, Germany) showed that the axial length was $15.38 \mathrm{~mm}$ in the right eye and $15.46 \mathrm{~mm}$ in the left eye. Indentation gonioscopy could not allow us to observe trabecular meshwork.

The patient was taking oral acetazolamide and the maximal topical hypotensive medications for her left eye. The visual fields determined by a Humphrey visual field analyzer SITA standard 24-2 program (Carl Zeiss Meditec AG) showed that the mean deviation (MD) was $-4.33 \mathrm{~dB}$ in her right eye and $-29.51 \mathrm{~dB}$ in the left eye.

The patient underwent surgery for bilateral primary angle-closure glaucoma with PEA + IOL implantation in her right eye and PEA + IOL implantation combined with trabeculotomy ab externo with metal trabeculotome in her left eye. After the surgery, the AC was still shallow, but the IOP stayed within the normal range for 9 months (Fig. 1). However, the IOP gradually increased and became uncontrollable at $38 \mathrm{mmHg}$ in the right eye and $33 \mathrm{mmHg}$ in the left eye with topical medications. We performed full-thickness scleral resection at the inferior two quadrants. The main purpose of this was expecting the prophylactic effect against uveal effusion caused by the next intraocular surgery. We also had a little expectation the scleral resection might be able to resolve this difficult condition. A thick sclera in nanophthalmic eye may lead to partial stenosis of the vortex veins, impairing normal venous outflow and causing overfilling of the choroid. This blood flow abnormality might be a cause of misdirection of aqueous humor. The pioneers of our ophthalmologists experienced some success after scleral resection [13, 14] for malignant glaucoma. Scleral resection is less invasive than intraocular surgery.

After failure of the scleral resection to the right eye, we undertook surgery on both eyes. This included performing an anterior vitrectomy through a clear cornea and creating a hole in the peripheral lens capsule combined with a peripheral iridectomy to both eyes. After creating a communication hole between the $\mathrm{AC}$ and posterior chamber, the $\mathrm{AC}$ deepened and the IOP decreased to within the normal range (Fig. 2). However, 2 months later, the AC of her left eye became shallow again and the IOP increased to $32 \mathrm{mmHg}$. Examination showed that the hole in the lens capsule was closed by Elschnig's pearls and we could not observe the trabecular meshwork with a gonio lens. We enlarged the iridectomy hole and lens capsule hole with a vitreous cutter through the AC. We also performed a trabeculectomy with mitomycin $\mathrm{C}$ at superior quadrant near peripheral capsulotomy and iridectomy to both eyes in order to reach the target IOP. At the last follow-up visit 4 years after the final trabeculectomy, the IOP was controlled at $12 \mathrm{mmHg}$ in both eyes without topical hypotensive medication.

Case 2 A 47-year-old woman had an acute angleclosure glaucoma attack in her left eye and received peripheral iridectomy in 2004. She was referred to the Hiroshima University Hospital for high IOP in both eyes in May 2010.

Her BCVA in the right eye was 20/25 with spherical $+9.00 \mathrm{D}$ and $+1.50 \mathrm{D}$ cylinder lens axis 100 . BCVA in her left eye was 20/25 with spherical $+9.00 \mathrm{D}$ and $+1.50 \mathrm{D}$ cylinder lens axis 80 .

She was receiving topical latanoprost once daily, timolol $0.5 \%$ and brinzolamide twice daily to both eyes. She had phakic in both eyes, and the IOP was $28 \mathrm{mmHg}$ in the right eye and $29 \mathrm{mmHg}$ in the left eye. The axial length of the right eye was $17.76 \mathrm{~mm}$ and in the left eye was $17.78 \mathrm{~mm}$ by ultrasound A-mode measurements (UD-800, Tomey Corporation, Nagoya, Japan), indicating nanophthalmic eyes. The angle structures could not be observed by gonioscopy even with indentation (Fig. 3). The MD of her right eye visual field was $-1.29 \mathrm{~dB}$ and in the left eye was $-15.31 \mathrm{~dB}$.

We performed sclerectomy and PEA + IOL implantation in her left eye. After cataract surgery, the AC slightly deepened, but the angle was still closed with extensive anterior synechia. The left eye IOP was $>20 \mathrm{mmHg}$. The anteriorly bulging peripheral iris that closed the angle, even with an open iridectomy hole, led us to diagnose the patient with misdirection syndrome. Three months after the initial surgery, an anterior vitrectomy was performed through the iridectomy hole and a peripheral lens 
Fig. 1 Case 1 Slit-lamp biomicroscopic photographs of the right phakic eye (a, b) and the left pseudophakic eye $(\mathbf{c}, \mathbf{d})$, showing a shallow anterior chamber even after cataract surgery in the left eye
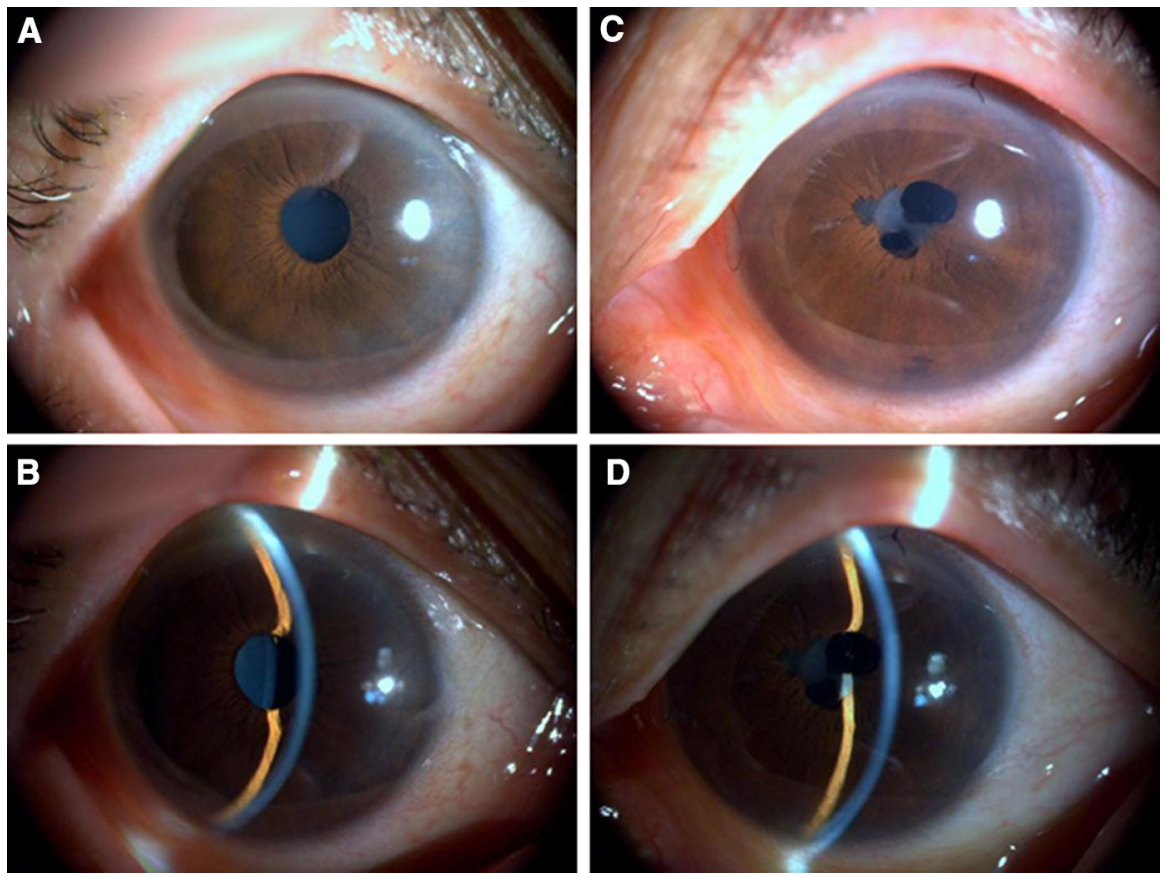

Fig. 2 Case 1 Slit-lamp biomicroscopic photographs of the right eye $(\mathbf{a}, \mathbf{b})$ and left eye $(\mathbf{c}, \mathbf{d})$, showing a normal anterior chamber in both eyes after lens capsulotomy and anterior vitrectomy
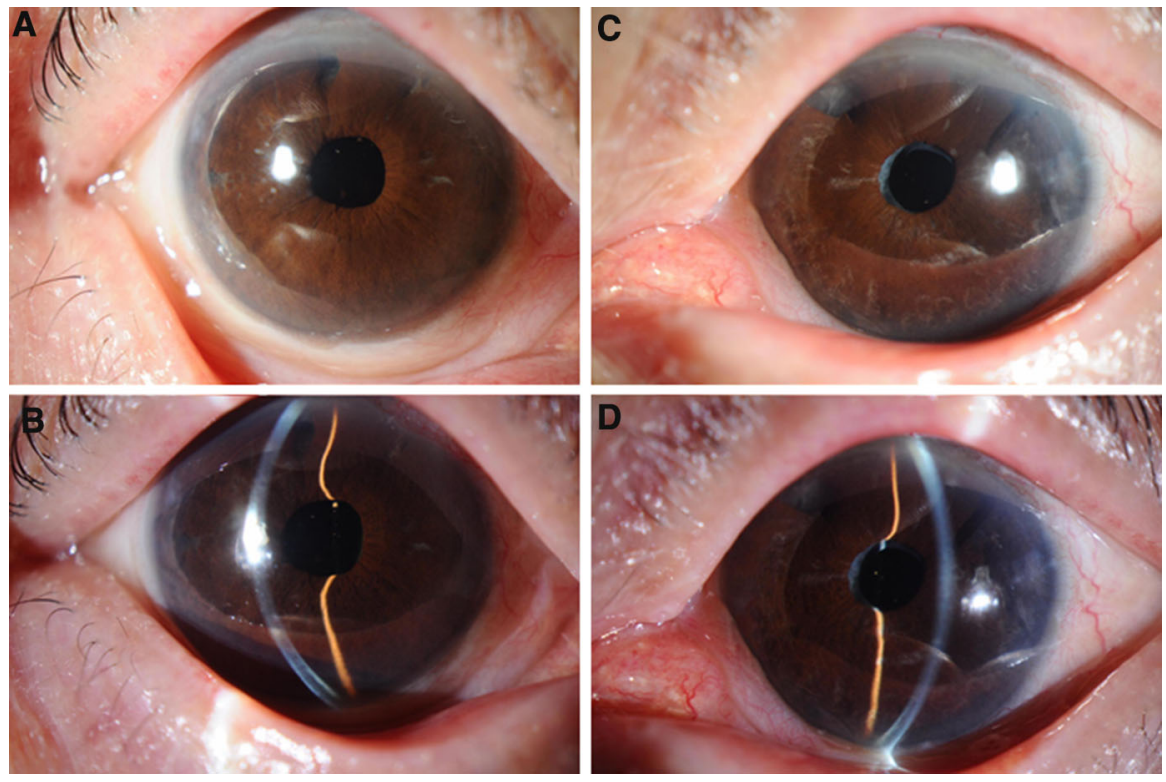

capsule hole through a clear cornea was created after failure to control the IOP with maximal anti-glaucoma medications. We also performed goniosynechialysis using a spatula under direct gonioscopy during the surgery. The IOP remained within normal limits from day 1 until 48 months after the anterior vitrectomy with daily latanoprost.
The patient was reluctant to have further surgery to her right eye, including laser iridoplasty or iridotomy, so we managed the IOP with topical medications. This was despite the patient having an elevated IOP in this eye for almost 3 years, and as a consequence, her visual field was degenerating. In April 2014, her right eye IOP increased to over $30 \mathrm{mmHg}$, which caused visual field damage ( $\mathrm{MD}=-1.29$ to $-14.81 \mathrm{~dB}$ ). 
Fig. 3 Case 2 Slit-lamp photographs of the right eye $(\mathbf{a}, \mathbf{b})$ and the left eye (c, d), showing a shallow anterior chamber in both eyes
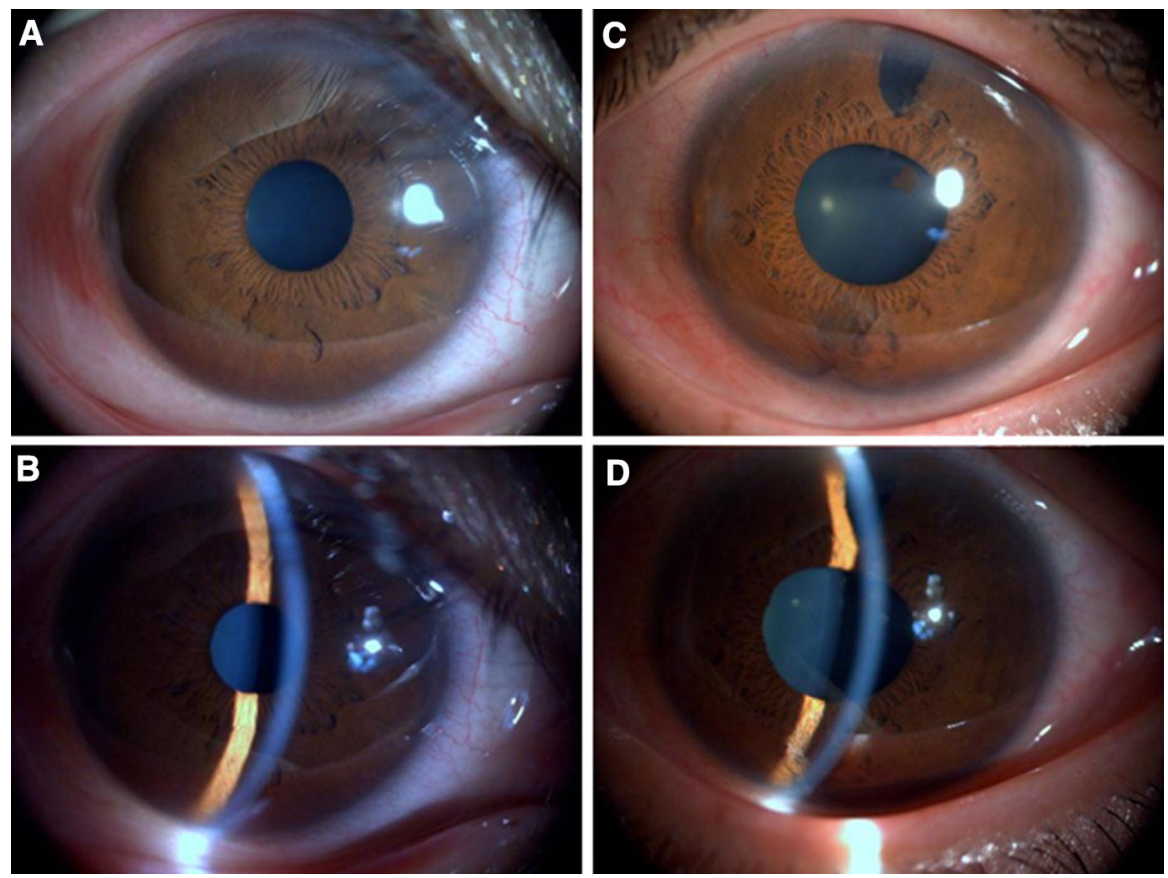

The patient agreed to have PEA + IOL implantation in her right eye. After successful cataract surgery, her right eye had a shallow AC with an IOP of $52 \mathrm{mmHg}$ and we diagnosed misdirection syndrome. We performed an anterior vitrectomy, capsulotomy and iridectomy through the AC combined with trabeculectomy as her iridocorneal touch continued for more than 4 years after her first visit. One day after surgery, her right eye IOP was $17 \mathrm{mmHg}$ with normal $\mathrm{AC}$ depth. Three days after the anterior vitrectomy and trabeculectomy, she developed uveal effusion with severe exudative retinal detachment. Two full-thickness scleral resections to the inferior quadrants between the rectal muscles resolved this condition. The final right eye IOP was $14 \mathrm{mmHg}$ and the left eye was $15 \mathrm{mmHg}$ at the last visit in December, 2015 (Fig. 4). The visual acuity in her right eye had decreased three lines compared with that at the first visit.

Case 3 A 44-year-old man with primary angleclosure glaucoma was referred to the Hiroshima University Hospital on December 2012.

His BCVA was 20/25 with spherical $+16.00 \mathrm{D}$ in the right eye and $20 / 32$ with spherical $+17.00 \mathrm{D}$ in the left eye at the first visit. The IOP was $27 \mathrm{mmHg}$ in the right eye and $17 \mathrm{mmHg}$ in the left eye. Slit-lamp biomicroscopy revealed that the AC was shallow, and computerized tomography showed the presence of a thickened sclera in both eyes (Fig. 5). Biometry revealed an axial length of $15.78 \mathrm{~mm}$ in the right eye and $15.84 \mathrm{~mm}$ in the left eye. We could not observe the trabecular meshwork with indentation gonioscopy.

The patient underwent scleral resection combined with PEA + IOL implantation in his right eye without any complications. The patient underwent scleral resection to prevent uveal effusion after cataract surgery. For the scleral resection, we made $2 \times 2 \mathrm{~mm}$ rectangular full-thickness scleral holes between the inferior rectal muscle and medial rectus muscle and the inferior rectal muscle and lateral rectus muscle.

One day after the surgery, his AC was still shallow and the IOP was $23 \mathrm{mmHg}$. The IOP gradually increased to $32 \mathrm{mmHg}$ as the $\mathrm{AC}$ continued to get shallower. Atropine eye drops and aqueous suppressant did not resolve the situation, and he was diagnosed with misdirection syndrome. One week after the cataract surgery, iridectomy, capsulotomy and anterior vitrectomy were performed through a clear cornea. The AC was finally opened and the IOP was stabilized. The BCVA improved to 20/20 with +15.00 spherical D, and the IOP was controlled at approximately $15 \mathrm{mmHg}$ with topical latanoprost, 
Fig. 4 Case 2 Slit-lamp biomicroscopic photographs of the right eye $(\mathbf{a}, \mathbf{b})$ and left eye $(\mathbf{c}, \mathbf{d})$, showing a deep anterior chamber in both eyes after lens capsulotomy and anterior vitrectomy
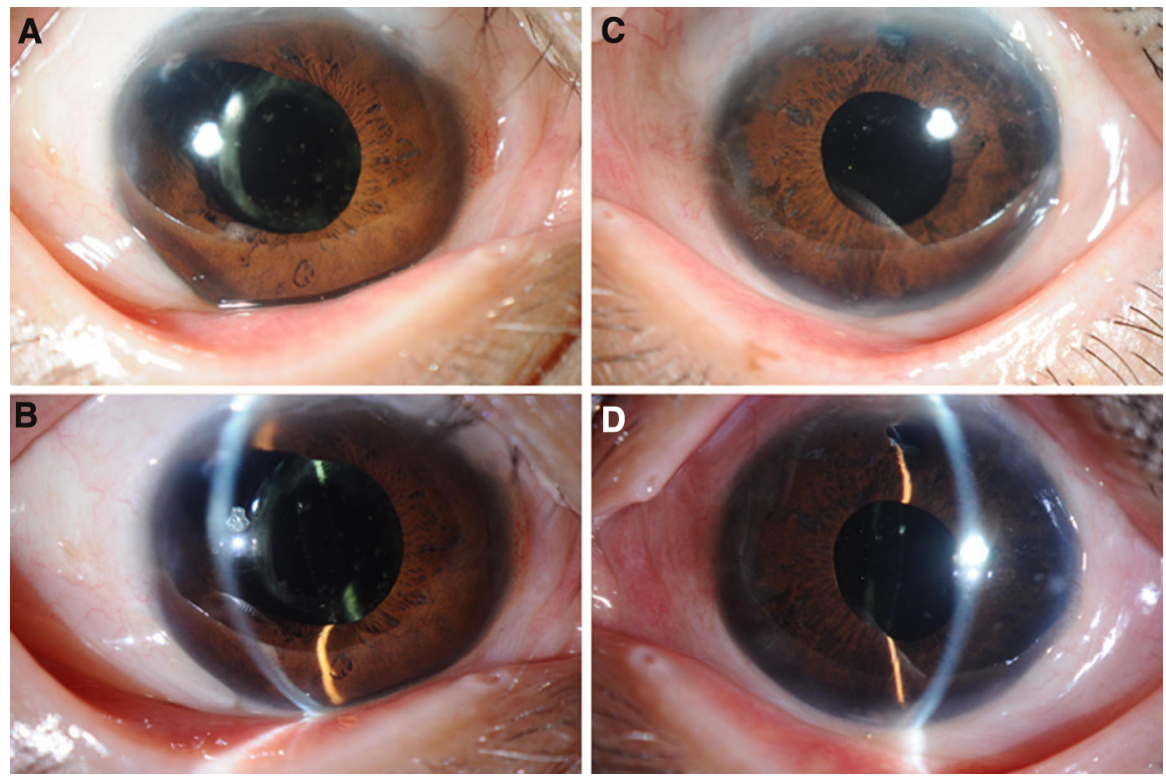

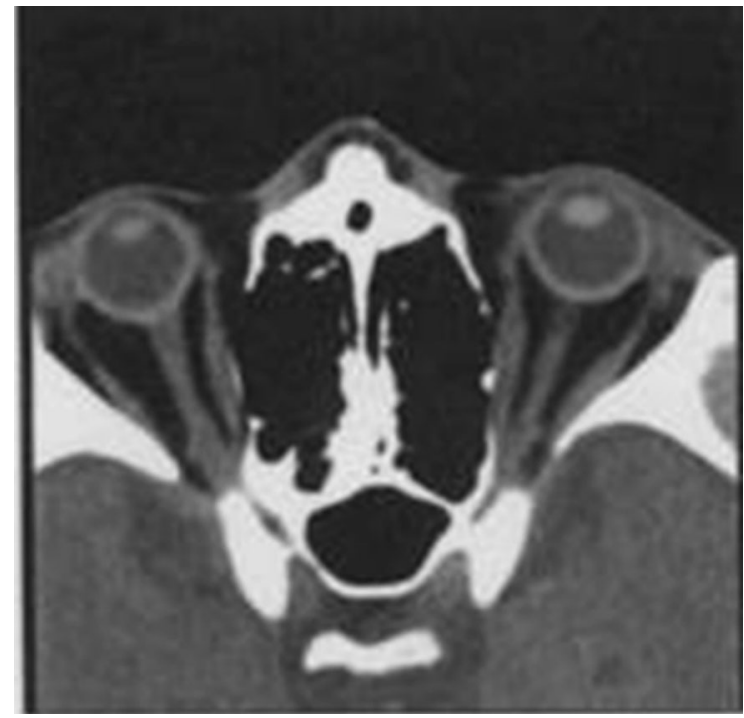

Fig. 5 Case 3 Computerized tomography showed the presence of a thickened sclera in both eyes

dorzolamide $1.0 \%$ and timolol $0.5 \%$. The IOP in the left eye was maintained in a normal range of 15-20 mmHg for 57 months after a laser iridotomy without any medication.

Case 4 A 69-year-old woman with bilateral primary angle-closure glaucoma was referred to the Hiroshima University Hospital on May 2014 with very shallow ACs and IOP over $30 \mathrm{mmHg}$ in her left eye.
Her BCVA was 20/50 with +15.00 spherical D in the right eye and 20/50 with +14.00 spherical $D$ and $-1.00 \mathrm{D}$ cylinder lens axis 90 in the left eye at the first visit. After laser iridotomy, the IOP decreased to $14 \mathrm{mmHg}$ in the right eye and $18 \mathrm{mmHg}$ in the left eye with latanoprost and brinzolamide $1.0 \%$. Slit-lamp biomicroscopy and anterior optical coherence tomography revealed very shallow ACs (Fig. 6), and ophthalmic ultrasonography showed no choroidal detachment in both eyes. The axial length was $16.50 \mathrm{~mm}$ in the right eye and $16.39 \mathrm{~mm}$ in the left eye.

PEA + IOL implantation was attempted on the left eye in July 2014. During surgery, the AC became shallow and we did not insert the IOL. The following day, the AC in the left eye remained shallow and the IOP was $20 \mathrm{mmHg}$. Topical atropine and aqueous inflow suppressant did not resolve the situation. One week later, a communication hole was created in the supra-nasal peripheral area between the anterior and posterior chambers with a vitreous cutter just before inserting the IOL. One day after surgery, the AC depth was normal and the IOP was $15 \mathrm{mmHg}$. The IOP and $\mathrm{AC}$ have remained stable for more than 2 years with no medication.

On August 2014, PEA + IOL implantation was performed without any complications on her right eye. One day after the surgery, the AC of the right eye was deep and the IOP was $14 \mathrm{mmHg}$, but 2 months post- 
Fig. 6 Case 4 Slit-lamp photographs of the right eye (a) and left eye (b), showing a shallow anterior chamber in both eyes. Anterior segment optical coherence tomographic image of the right eye (c) and left eye (d), showing a shallow anterior chamber in both eyes before surgery
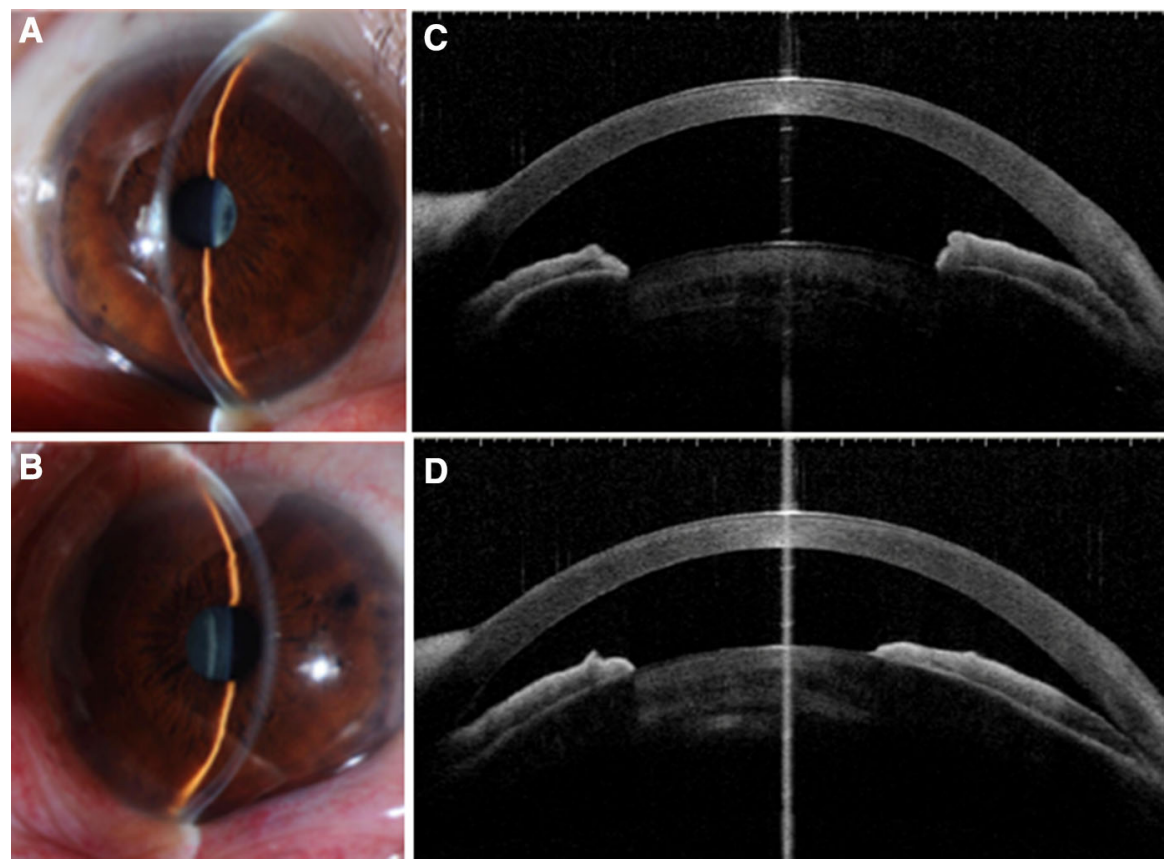

surgery, the AC became shallow and the IOP of the right eye increased to $32 \mathrm{mmHg}$. This situation was not resolved with glaucoma medication and topical atropine. From the AC configuration and anterior segment optical coherence tomography findings, we diagnosed her as having misdirection syndrome in. An anterior iridectomy, peripheral capsulotomy and anterior vitrectomy were performed with a vitreous cutter on October 2014. On the first postoperative day, the misdirection syndrome was resolved, the AC was deepened, and the IOP was $15 \mathrm{mmHg}$ in the right eye. After a follow-up period of 20 months, examination with optical coherence tomography showed that the AC was deep in both eyes (Fig. 7). The IOP remained at around $14 \mathrm{mmHg}$ without medication in both eyes.

\section{Discussion}

The term malignant glaucoma was coined by Von Graefe in 1869 [13]. He noted that a number of patients had a shallowing of the AC together with high IOP after peripheral iridectomy for acute angleclosure glaucoma [13]. The exact pathophysiology of the high IOP has not been determined, but alterations in the ciliary body, choroid, lens, zonules and vitreous have been suggested as the mechanism.
These alterations can cause a diversion of the aqueous humor into the vitreous cavity [14]. Abnormal anatomical relationships among the structures in the anterior segment can lead to disruptions in the direction of aqueous humor flow. For example, a relatively large crystalline lens can easily block the normal aqueous flow from the posterior chamber to the AC [15]. A thick sclera may lead to a partial stenosis of the vortex veins, which would impair the normal venous outflow, causing an overfilling of the choroid. This choroidal thickness abnormality may be a cause of the misdirection of aqueous humor [14].

The management of misdirection syndrome is complicated. The first line of treatment should be medical management including topical cycloplegia, aqueous suppressants and hyperosmotic agents [16]. The goal of medical management is to decrease aqueous humor production, shrink the vitreous body and move the iris-lens diaphragm posteriorly [16]. YAG laser photocoagulation photodisruption can be used to create a hole in the anterior hyaloid membrane, and argon laser irradiation of the ciliary processes can shrink the ciliary processes, resulting in disruption of the ciliary block $[17,18]$. If medical or laser treatments fail to resolve the misdirection syndrome, surgery should be considered. Surgical decompression of the vortex veins or full-thickness sclerectomy is 
Fig. 7 Case 4 The anterior chamber is deep as confirmed by the optical coherence tomography examination of the right eye (a) and left eye (b)
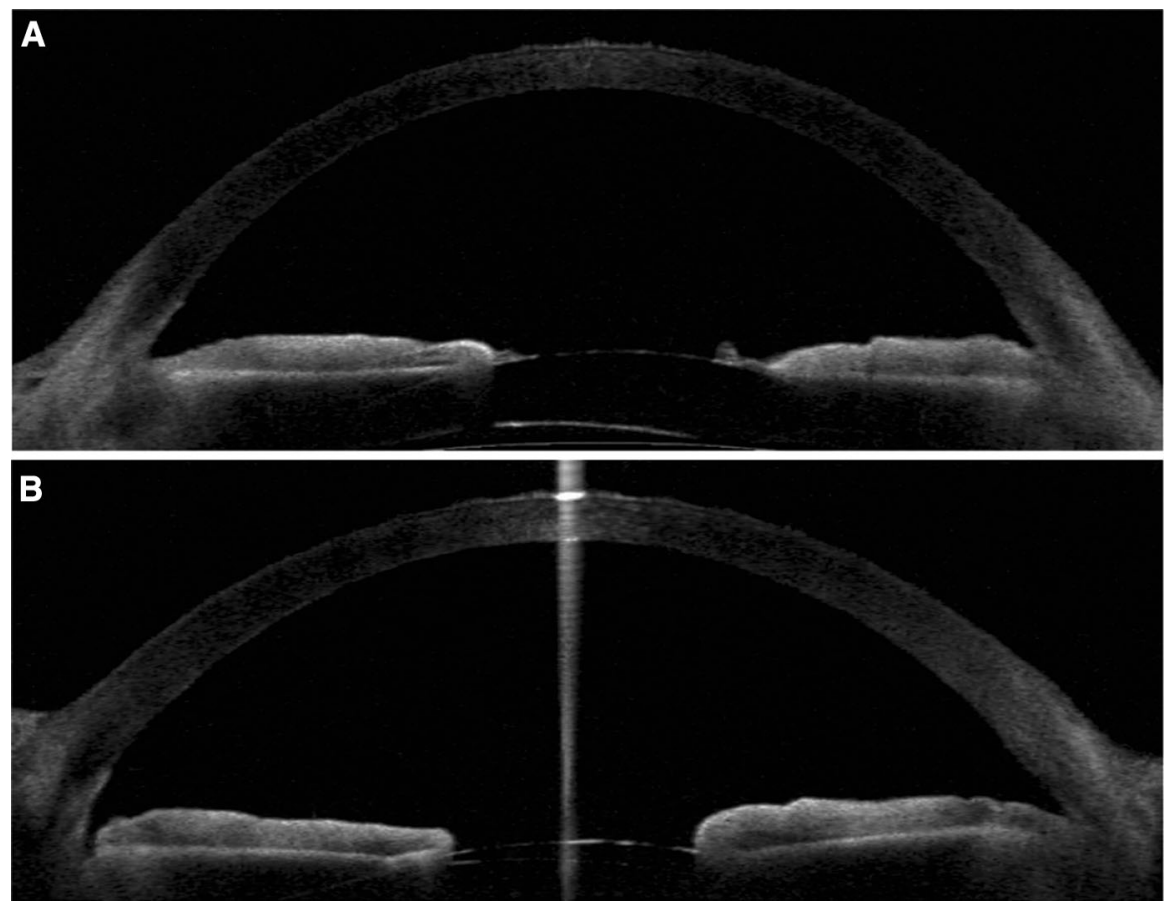

recommended by some authors for misdirection syndrome in nanophthalmic eyes [19]. However, in this study scleral resection of both eyes of Case 1 and the left eye of Case 2 did not resolve the misdirection condition and Case 3 developed misdirection syndrome after scleral resection. We conclude that scleral resection is not effective in resolving or preventing the development of misdirection syndrome in nanophthalmic eyes. Conversely, we did demonstrate that scleral resection was effective for the treatment of uveal effusion.

Pars plana vitrectomy has been reported to be effective for resolving misdirection syndrome [20]. However, Hosoda et al. [21] reported two cases in which misdirection syndrome recurred after complete vitrectomy. They performed an emergency iridectomy and local zonulectomy using a 25-gauge vitreous cutter and achieved effective results. Byrnes et al. [22] reported persistent or recurrent ciliary block glaucoma in $50 \%$ of phakic patients and in $10 \%$ of pseudophakic eyes in their series of patients that underwent pars plana vitrectomy for misdirection syndrome. The posterior diversion of the aqueous humor by a ciliovitreo-lenticular block caused an unexpected anterior hyaloid clog between the ciliary processes. This anteriorly compressed vitreous is pressed into the folds of the ciliary processes and causes an alteration of the permeability of the aqueous, leading to a marked rise in the IOP. This clogging between the ciliary processes is difficult to remove (Hirota A, Miyoshi T, ESCRS Film Festival Grand Prize, 2013).

The structure of the anterior segment of nanophthalmic eyes is different from that of eyes with angle closure [1]. In a study by Ohkita et al. [23], the authors created three holes in the peripheral retina while making three ports for a vitrectomy to treat a retinal detachment in a nanophthalmic eye. The region $3.5 \mathrm{~mm}$ from the limbus was not the pars plana but rather the retina in their small eye. The length of the pars plana is very short in nanophthalmic eyes. [23] To avoid this complication during vitrectomy, we performed an anterior vitrectomy through the clear cornea as a safe surgical procedure in patients with nanophthalmos. This approach also has the advantage of avoiding the pars plicate area where the anterior vitreous is packed during misdirection syndrome. However, when the communication hole in the lens capsule or iris is closed, the IOP will increase associated with a shallowing of the AC. We recommend making a large enough opening to prevent it from being closed by Elschnig's pearls. 
The results in Cases 1 and 2 showed that maintaining normal aqueous flow is insufficient to obtain normal IOP control when the angle is extensively closed. We recommend a trabeculectomy to address the high IOP caused by severe peripheral anterior synechia, as it may be beneficial to correct the misdirection of aqueous flow before confronting the severe angle synechia.

Our study has some limitations. We showed the effectiveness of creating a hole in the lens capsule and performing an anterior vitrectomy through the AC for misdirection syndrome in seven nanophthalmic eyes from four patients. Our experience is unlikely apply to all misdirection syndrome cases in nanophthalmos as the pathological mechanism of misdirection syndrome is not fully understood. As nanophthalmic eyes can show unexpected reactions to surgical procedures and the frequency of nanophthalmos is low, it is difficult to conduct a large-scale randomized prospective study to find out the best countermeasure for misdirection syndrome in nanophthalmos. We must collect more information on the optimal procedures to improve treatment of this difficult condition.

In conclusion, creating a communication hole between the anterior and posterior chambers through the cornea can be a beneficial option to resolve misdirection syndrome in patients with nanophthalmos. Surgical interventions on nanophthalmic eyes can cause many unexpected complications, and the surgeon must know how to prevent and resolve these complications.

Acknowledgements We wish to thank Professor Emeritus Duco Hamasaki of the Bascom Palmer Eye Institute of Miami, Florida, for his discussions on this study. We thank David Dimasi, Ph.D., from Edanz Group (www.edanzediting.com/ac) for editing a draft of this manuscript.

\section{Compliance with ethical standards}

Conflict of interest The authors declare that they have no conflict of interest.

Ethical approval All procedures performed in studies involving human participants were in accordance with the ethical standards of the Institutional Review Board of the Hiroshima University Hospital, Hiroshima, Japan and with the 1964 Helsinki Declaration and its later amendments or comparable ethical standards.

Informed consent Informed consent was obtained from all individual participants included in the study.
Open Access This article is distributed under the terms of the Creative Commons Attribution 4.0 International License (http:// creativecommons.org/licenses/by/4.0/), which permits unrestricted use, distribution, and reproduction in any medium, provided you give appropriate credit to the original author(s) and the source, provide a link to the Creative Commons license, and indicate if changes were made.

\section{References}

1. Ritch R, Lowe RF (1996) Angle closure glaucoma: clinical types. In: Ritch R, Shields MB, Krupin T (eds) The glaucomas. Mosby, St. Louis, pp 831-833

2. Ryan EA, Zwaan J, Chylack LT Jr (1982) Nanophthalmos with uveal effusion: clinical and embryologic considerations. Ophthalmology 89:1013-1017

3. Day AC, MacLaren RE, Bunce C, Stevens JD, Foster PJ (2013) Outcomes of phacoemulsification and intraocular lens implantation in microphthalmos and nanophthalmos. J Cataract Refract Surg 39:87-96

4. Utman SA (2013) Small eyes big problems: is cataract surgery the best option for the nanophthalmic eyes? J Coll Physicians Surg Pak 23:653-656

5. Moghimi S, Lin S (2011) Role of phacoemulsification in angle closure glaucoma. Eye Sci 26:121-131

6. Trikha S, Perera SA, Husain R, Aung T (2015) The role of lens extraction in the current management of primary angleclosure glaucoma. Curr Opin Ophthalmol 26:128-134

7. Feng YF, Wang DD, Zhao YE, Li JH, Savini G, Huang JH (2013) Surgical management of malignant glaucoma with white cataract in nanophthalmos. J Cataract Refract Surg 39:1774-1777

8. Brockhurst RJ (1974) Nanophthalmos with uveal effusion: a new clinical entity. Trans Am Ophthalmol Soc 72:371-403

9. Moradian S, Kanani A, Esfandiari H (2011) Nanophthalmos. J Ophthalmic Vis Res 6:145-146

10. Dave P, Senthil S, Rao HL, Garudadri CS (2013) Treatment outcomes in malignant glaucoma. Ophthalmology 120:984-990

11. Bitrian E, Caprioli J (2010) Pars plana anterior vitrectomy, hyaloido-zonulectomy, and iridectomy for aqueous humor misdirection. Am J Ophthalmol 150:82-87

12. Wu W, Dawson DG, Sugar A, Elner SG, Meyer KA, McKey JB, Moroi SE (2004) Cataract surgery in patients with nanophthalmos: results and complications. J Cataract Refract Surg 30:584-590

13. Ruben S, Tsai J, Hitchings RA (1997) Malignant glaucoma and its management. Br J Ophthalmol 81:163-167

14. Luntz MH, Rosenblatt M (1987) Malignant glaucoma. Surv Ophthalmol 32:73-93

15. Singh OS, Simmons RJ, Brockhurst RJ, Trempe CL (1982) Nanophthalmos: a perspective on identification and therapy. Ophthalmology 89:1006-1012

16. Shen CJ, Chen YY, Sheu SJ (2008) Treatment course of recurrent malignant glaucoma monitoring by ultrasound biomicroscopy: a report of two cases. Kaohsiung J Med Sci 24:608-613 
17. Herschler J (1980) Laser shrinkage of the ciliary processes. A treatment for malignant (ciliary block) glaucoma. Ophthalmology 87:1155-1159

18. Epstein DL, Steinert RF, Puliafito CA (1984) NeodymiumYAG laser therapy to the anterior hyaloid in aphakic malignant (ciliovitreal block) glaucoma. Am J Ophthalmol 98:137-143

19. Simmons RJ (1972) Malignant Glaucoma. Br J Ophthalmol 56:263-272

20. Meng L, Wei W, Li Y, Hui X, Han X, Shi X (2015) 5-Gauge pars plana vitrectomy for ciliary block (malignant) glaucoma. Int Ophthalmol 35:487-493
21. Hosoda Y, Akagi T, Yoshimura N (2014) Two cases of malignant glaucoma unresolved by pars plana vitrectomy. Clin Ophthalmol 8:677-679

22. Byrnes GA, Leen MM, Wong TP, Benson WE (1995) Vitrectomy for ciliary block (malignant) glaucoma. Ophthalmology 102:1308-1311

23. Ohkita T, Emi K, Toyoda E, Ueno C, Sawada K, Sawada K, Matsumura N, Morita S, Kashimoto D, Oyagi T, Ikeda T (2008) Efficacy of vitreous surgery for uveal effusion syndrome. Nippon Ganka Gakkai Zasshi 112:472-475 